\title{
Role of MicroRNAs in Hepatic Fibrosis Progression
}

\author{
Devaraj Ezhilarasan
}

Biomedical Research Unit and Laboratory Animal Centre, Saveetha Dental College and Hospitals, Saveetha Institute of Medical and Technical Sciences, Chennai, Tamil Nadu, 600 077, India.

\section{ARTICLE INFO \\ Article history: \\ Received on: 14/02/2018 \\ Accepted on: 19/04/2018 \\ Available online: 30/05/2018}

Key words:

Hepatic stellate cells,

extracellular matrix,

miRNAs, liver fibrosis.

\begin{abstract}
Acute liver injury induced deviation in liver architecture are transient and often reversible. While chronic liver diseases lead to progressive net accumulation of extracellular matrix (ECM) in the liver leading to hepatic fibrosis. If hepatic fibrosis is untreated, hepatic fibrosis can lead to cirrhosis and consequence of organ failure and death. Hepatic stellate cells (HSCs) activation plays a significant role in the pathogenesis of hepatic fibrosis. MicroRNAs are short, non-coding, single-stranded RNA and their modulation upon chronic liver injury are said to involve in the activation and proliferation of HSCs that causes synthesis and accumulation of the enormous amount of ECM proteins by HSCs in liver hepatic fibrosis consequently. This review summarizes the modulation of key miRNAs pertaining to hepatic fibrosis.
\end{abstract}

\section{INTRODUCTION}

Epidemiological studies revealed that chronic liver disease (CLD) is one of the important sources of health and economic burden worldwide. According to the Global Burden of Disease Study in 2010, about 1.75 million deaths were attributable to CLD regardless of any etiology (Udompap et al., 2015). The chronic liver disease is responsible for 2 million deaths annually. The main etiological factors associated with this mortality were found to be alcoholic liver disease and hepatitis B and C viral infections (Udompap et al., 2015). Hepatic fibrosis is one of the common pathological sequel of all CLD and responsible for significant morbidity and mortality worldwide (Ezhilarasan et al., 2017).

\section{Hepatic fibrosis}

Hepatic fibrosis is commonly preceded by CLD and result from the progressive accumulation and decreased

${ }^{*}$ Corresponding Author

D. Ezhilarasan Ph.D., Assistant Professor, Department of

Pharmacology, Biomedical Research Unit and Laboratory Animal

Centre, Saveetha Dental College and Hospitals, Saveetha Institute of

Medical and Technical Sciences, Chennai, Tamil Nadu, 600 077, India.

E-mail:ezhild@gmail.com degradation or remodeling of the extracellular matrix (ECM), any etiology, including viral infection, alcoholic liver disease and nonalcoholic steatohepatitis (Ezhilarasan et al., 2015). In response to chronic liver injury, hepatic stellate cells (HSCs) undergo transdifferentiation of quiescent into contractile, proliferative and highly activated myofibroblast (MFB) like phenotype and this phenotypic activation is demonstrated in experimental and clinical studies and is considered as central event of hepatic fibrosis (Tsuchida and Friedman, 2017; Ezhilarasan et al., 2016; Ezhilarasan et al., 2012). If hepatic fibrosis is untreated, hepatic fibrosis causes distortion of liver architecture leading to organ contraction, nodular formation, liver cirrhosis, and hepatocellular carcinoma (HCC) and ultimately death (Jung and Yim, 2017).

\section{MicroRNAs and chronic liver diseases}

MicroRNAs (miRNA or miR) are short, 18 to 25 nucleotide long, non-coding, single-stranded RNA that function as regulatory molecules and said to involve in a series of vital processes including cell growth and differentiation, apoptosis, metabolism and the pathogenesis of various human diseases (Kitano and Bloomston, 2016). A single miRNA is shown to regulate several genes and vice versa. Previous studies have demonstrated the significant role of miRNA in liver diseases (Hayes and Chayama, 2016). It has been mentioned that even a 
small alteration in miRNA expression may significantly affect the battery of gene expressions and thus alter the transcriptome (Kerr et al., 2011). In liver, the interference of miRNA expressions has been implicated in varieties of CLD.

miRNA expression is considered tissue or a specific organ. For instance, in liver, miR-122 is one of the most abundant in hepatocytes $(\sim 70 \%)$. miRNAs present outside the hepatocytes such as in neutrophils, HSCs are also implicated for their role in CLD. For instance, miR-22 is an important regulator to block neutrophil infiltration in alcoholic liver diseases (ALD) (Li et al., 2016). Studies have also documented that miR-122, 155, 34a, 21, $146 \mathrm{a}$ and $125 \mathrm{~b}$ are increased in the circulation of CLD patients (Shigehara et al., 2011; Weber et al., 2010).

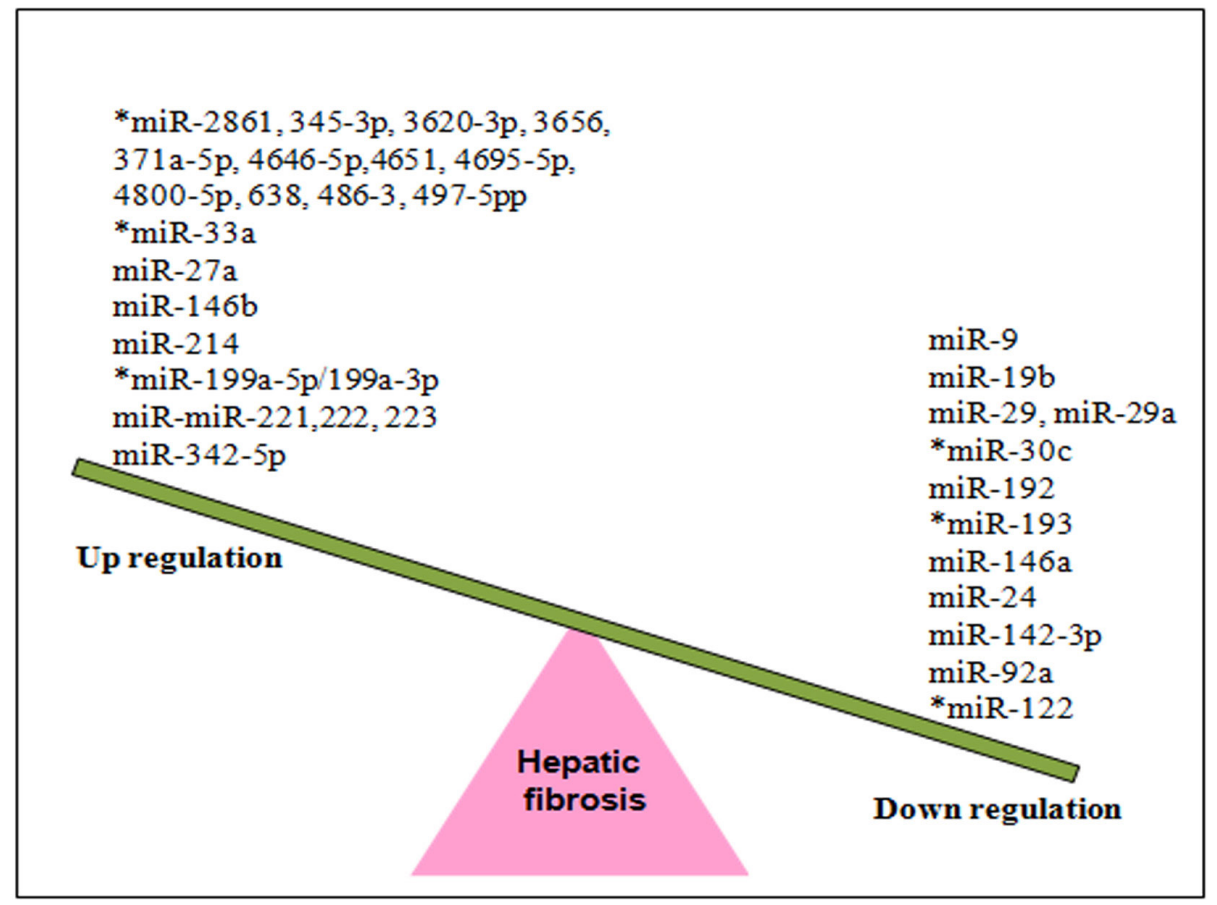

Fig. 1: Modulation of some of the key miRNAs in liver during the progression of hepatic fibrosis. *clinical studies.

\section{Role of microRNAs in hepatic fibrosis}

As mentioned before, HSCs play a significant role in the progression and onset of hepatic fibrosis. Therefore, studies have identified HSCs specific miRNAs that are responsible for early events of hepatic fibrosis (Figure 1). The first miRNA expression profile in human HSCs was reported by Coll et al. (2015). They have identified key miRNAs pertain to the maintenance of the quiescent phenotype of HSCs. It is shown that miR-192 downregulation during the activation of qHSCs in experimental studies and that over expression of miR-192 found to have a key role in the HSCs activation by reducing the perpetuating potentials of HSCs (Coll et al., 2015).

\section{Role of miRNAs in TGF- $\beta$ signaling and ECM synthesis}

Transforming growth factor beta (TGF- $\beta$ ) is a profibrogenic cytokine that drives hepatic fibrosis by inducing HSC proliferation and ECM production (Xu et al., 2016). In $\mathrm{CCl}_{4}$-induced hepatic fibrosis mice model, miR-29 mediates the regulation of liver fibrosis. miR-29 is said to regulate TGF- $\beta$ - and nuclear factor- $\kappa \mathrm{B}(\mathrm{NF}-\kappa \mathrm{B})$-dependent decrease of miR-29 family members in HSC with consequent increase of ECM related gene expressions. The above findings were well correlated with clinical studies in which patients with advanced liver cirrhosis showed significant decrease in miR-29a levels in their serum than that of normal subjects or patients with early fibrosis (Roderburg et al.,
2011) Further, it has been identified that miR-30c and miR-193 are also responsible for TGF- $\beta$-dependent regulation of controlling ECM genes expressions in $\mathrm{CCl}_{4}$-induced fibrosis model. The miR-30c and miR-193 have been shown to downregulate during the hepatic fibrosis in experimental and human liver (Roy et al., 2015). These studies clearly indicate the fact that miR-29a, miR-30c, and miR-193 have the predominant role in the TGF- $\beta$ mediated ECM related gene expressions and activation of HSCs and hepatic fibrosis.

It is a well-established fact that TGF- $\beta$ is one of the important fibrogenic cytokine responsible for the activation of quiescent HSCs. Inhibitory effect of miR-19b in vitro analyses of HSCs has been reported. TGF- $\beta$ signaling is inhibited by the miR$19 \mathrm{~b}$. It was observed that the downregulation of miR-19b leading to activation of HSCs while it's upregulation shows concomitant fibrosis regression in rat liver. Similarly, miR-19b expression was markedly downregulated in the onset of hepatic fibrosis in human (Lakner et al., 2012). The miR-146a is shown to downregulate in liver fibrotic tissues and it was suggested as a novel regulator to modulate HSC activation during TGF- $\beta 1$ induction by targeting SMAD4 (He et al., 2012). Regulation of miR-33a expression in liver tissue was also implicated in the hepatic fibrosis. Particularly, miR-33a expression increased only in HSCs and not in other fibrosis associated cells such as Kupffer cells, hepatocytes etc. The upregulation of miR-33a expression activates the TGF- $\beta 1$ 
signalling in HSCs (Huang et al., 2015). Ge et al. (2014) reported that modulation of 48 miRNAs was found in porcine seruminduced hepatic fibrotic rats. Among 48 miRNAs the expressions levels of miR-27a and miR-146b have been significantly increased with concomitant increase in fibrotic marker gene expressions such as TGF- $\beta$ and collagen 1 . The functional role of miR-9 was investigated in a $\mathrm{CCl}_{4}$-induced mouse model of liver fibrosis. Interestingly, miR-9 level was found to be downregulated in fibrotic liver tissue and in activated HSCs. miR-9 limits liver fibrosis by controlling the perpetuating nature of activated HSCs by directly targeting multidrug resistance-associated protein 1 (MRP1/ABCC1) (Sun et al., 2017). Inhibition of miR-24 has directly increased the TGF- $\beta 1$ expression consequently increasing hepatic fibrosis (Hall et al., 2017).

Moreover, the profibrogenic role of miR-214 has been previously studied using transgenic mouse model (PDGF-C TG) in which platelet-derived growth factor $\mathrm{C}$ (PDGF-C) is over expressed resulting in hepatic fibrosis. The upregulation of miR-214 has been well correlated with the progression of hepatic fibrosis in PDGF-C TG mice. The miR-214 was found to involve in the progression of liver fibrosis by modulating the TGF- $\beta$ related signaling pathways. Therefore, locked nucleic acid (LNA)-anti-miR-214 was injected into PDGF-C TG mice to evaluate the fibrosis regression. Interestingly, LNA-anti-miR-214 treatment significantly reduced the profibrotic gene expressions such as collagen $1 \mathrm{a} 2$ and $4 \mathrm{a} 1$, TGF- $\beta 1$ and $\alpha$-smooth muscle actin ( $\alpha$-SMA) with a concomitant decrease in the levels of $\mathrm{p}$-extracellular signal-regulated kinases $1 / 2$ and p-AKT (Okada et al., 2015). It was also found that upregulation of miR-199a-5p/199a-3p and miR-221/222 in the human liver in a fibrosis progression-dependent manner. In particular, miR-221/222 was upregulated in LX-2 cells (human hepatic stellate cell line), also increased during the course of culture-dependent activation of mouse primary HSCs and also increased in the experimental mouse models of liver fibrosis (Ogawa et al., 2012). This study demonstrated the upregulation of miR-221/222 in primary HSCs and immortalized HSCs cell line and even in experimental models.

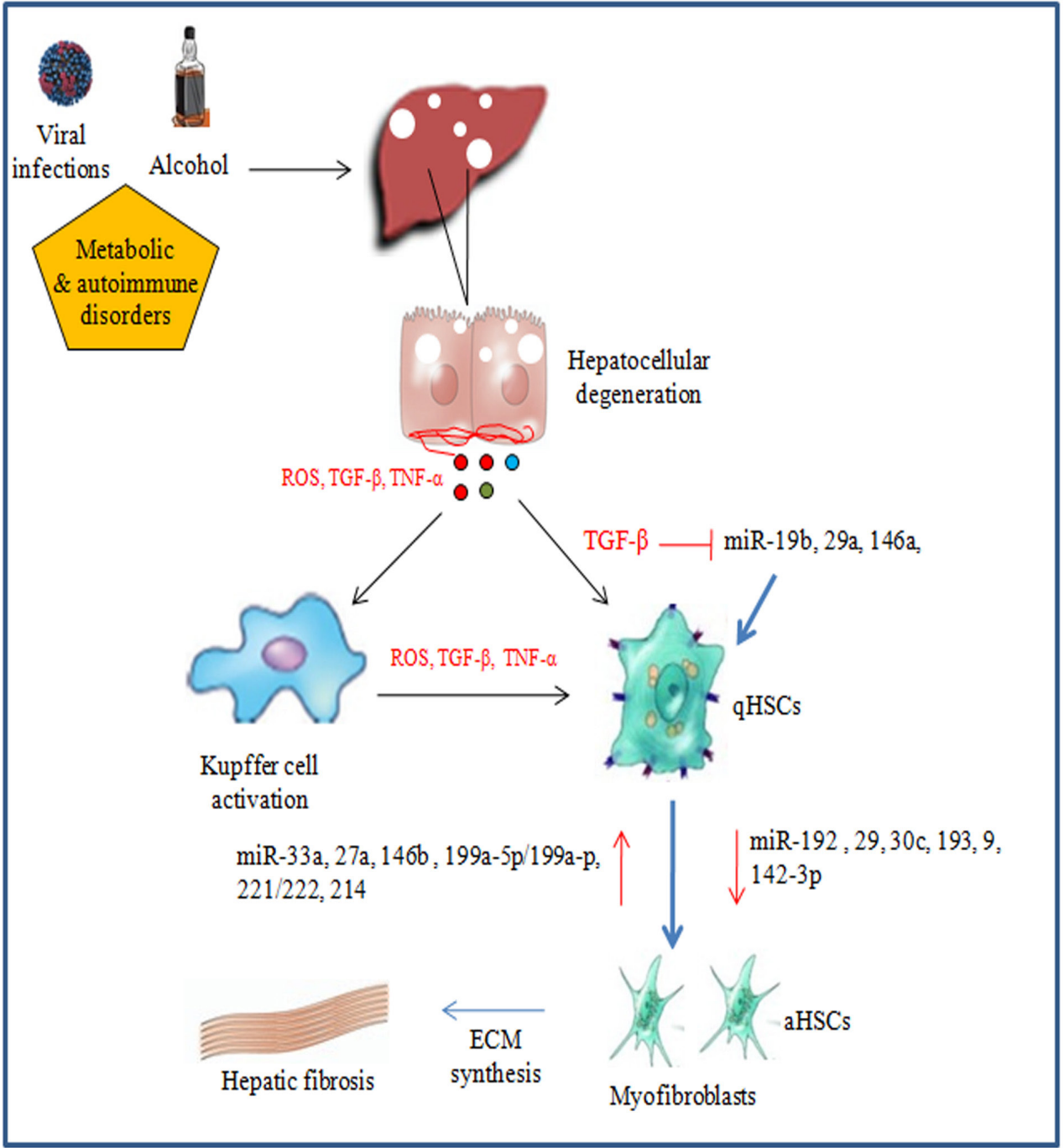

Fig. 2: Role of miRNAs in the progression of hepatic fibrosis due to various etiology. ROS-reactive oxygen species; TGF- $\beta$ - Transforming growth factor- $\beta$; TNF- $\alpha$ tumor necrosis factor- $\alpha$; qHSCs - Quiescent hepatic stellate cells; aHSCs - Activ atedhepatic stellate cells, ECM-Extracellular matrix.

In a very recent study, leptin, an adipocyte-derived hormone has been reported to decrease the sterol regulatory element-binding protein-1c (SREBP-1c) and promotes the activation the HSCs. Further, miR-122 inhibited leptin-induced 
liver fibrosis in a leptin-deficient mouse model (Zhai et al., 2017). The miR-142-3p has recently identified as a novel regulator of activation of HSCs. The miR-142-3p level has been shown to reduce in the activated HSCs. Therefore, HSCs was transfected with mir-142-3p and studied for its effects against activated HSCs. This study reports that ectopic expression of miR-142-3p in activated HSCs caused a decrease in proliferation and viability and blocked HSCs activation (Yang et al., 2017).

\section{miRNAs and human hepatic fibrosis}

In a recent study, miRNA expression pattern has been evaluated in liver tissue collected from the 40 chronic hepatitis $\mathrm{B}$ virus (HBV) associated hepatic fibrosis patients at stage S0-4. There were around 105 different miRNAs were found to modulated significantly in fibrotic tissues (Chen et al., 2017a). In HBV associated hepatic fibrosis and cirrhotic patients, miR-30 has been directly regulated the expression of IL-6R, one of the potent pleiotropic cytokine that regulates cell growth and differentiation (Chen et al., 2017b). In clinical studies, the paradigm of 43 hepatic miRNAs in NAFLD fibrosis model was reported previously. This study shows that differential expression of miR-17, miR-31, miR150, miR-182, miR-183, miR-219a, miR-224, miR-378c, miR$378 \mathrm{i}$, and miR-590 (Leti et al., 2015). Schematic role of miRNAs in the activation of HSCs and onset of hepatic fibrosis is depicted in Figure 2.

\section{Circulating miRNAs for early diagnosis of liver fibrosis}

miRNAs released into the systemic circulation upon hepatocellular damage and have shown as a promising new class of tissue-specific biomarkers (Krauskopf et al., 2017). Development of such a reliable non-invasive serum miRNA biomarkers may provide early detection of liver fibrosis and therefore studies have reported the modulation of miRNA expression in the circulation during the onset of hepatic fibrosis (Enache et al., 2014). The miR-223 expression is shown to dysregulated in liver tissue of mice after induction of liver fibrosis. The levels were also found to concomitantly express highly in the serum during the fibrosis progression. The modulation of miR-223 has also been confirmed in clinical studies in which it was correlated with liver cirrhosis patients (Schueller et al., 2017). The miR-122 levels found to decrease in circulation of patients with severe fibrosis due to hepatitis $\mathrm{B}$ and $\mathrm{C}$ virus infection and loss of liver cells were implicated in the decrease observed in the miR-122 in circulation during the fibrosis progression (Nakamura et al., 2017; Trebicka et al., 2013). This observation shows that analysis of miR-122 in serum could be one of the possible markers for early diagnosis of hepatic fibrosis due to hepatitis virus infections. In another study, plasma level of miR-142-3p significantly decreased in patients with hepatic cirrhosis (Yang et al., 2017). Undoubtedly, these studies show that circulating microRNAs can be potential and emerging biomarkers for early diagnosis of CLD.

\section{CONCLUSION}

During the progression of hepatotoxicity, a variety of signaling pathways triggered by proinflammatory factors and profibrogenic cytokines involving in the process of hepatic fibrosis. Dysregulation of miRNAs has been implicated in the onset of hepatic fibrosis. Following chronic liver injury modulation of miRNAs triggered hepatic fibrosis is associated with a) direct activation, proliferation, and migration of HSCs, b) expression of several profibrogenic cytokines such as TGF- $\beta$ and PGDF etc., c) high expressions of genes responsible for ECM synthesis such as different types of collagens. Since miRNA modulation has the myriad role in the activation of quiescent HSCs into myofibroblasts; miRNAs may represent novel therapeutic targets for the strategies of treatment of hepatic fibrosis.

\section{CONFLICTS OF INTEREST}

There are no conflicts of interest.

\section{FINANCIAL SUPPORT AND SPONSORSHIP}

Nil.

\section{REFERENCES}

Chen R, Wu JC, Liu T, Qu Y, Lu LG, Xu MY. MicroRNA profile analysis in the liver fibrotic tissues of chronic hepatitis B patients. J Dig Dis. 2017a; 18(2):115-124.

Chen Y, Yang S, Peng Y, Yang Z. The regulatory role of IL-6R in hepatitis B-associated fibrosis and cirrhosis. Braz J Med Biol Res. 2017b; 50(11):e6246.

Coll M, E Taghdouini A, Perea L, Mannaerts I, Vila-Casadesús M, Blaya D, et al. Integrative miRNA and Gene Expression Profiling Analysis of Human Quiescent Hepatic Stellate Cells. Sci Rep. 2015; $5: 11549$.

Enache LS, Enache EL, Ramière C, Diaz O, Bancu L, Sin A, et al. Circulating RNA molecules as biomarkers in liver disease. Int J Mol Sci. 2014; 15(10):17644-66.

Ezhilarasan D, Lakshmi T, Karthikeyan S. In vivo experimental models for hepatotoxin induced fibrosis-A toxicological view. Toxicol Int. $2015 ; 22(3): 1-9$.

Ezhilarasan D, Evraerts J, Sid B, Pedro Buc-Calderon, Karthikeyan S, Sokal E, et al. Silibinin induces hepatic stellate cell cycle arrest via enhancing p53/p27 and inhibiting Akt downstream signaling protein expression. Hepatobiliary Pancreat Dis Int. 2017; 16(1):80-87.

Ezhilarasan D, Evraerts J, Sid B, Pedro Buc-Calderon, Karthikeyan S, et al. Silibinin inhibits proliferation and migration of human hepatic stellate LX-2 cells. J Clin Exp Hepatol. 2016; 6(3):167-174.

Ezhilarasan D, Karthikeyan S, Vivekanandan P. Ameliorative effect of silibinin against $\mathrm{N}$-nitrosodimethylamine-induced hepatic fibrosis in rats. Environ Toxicol Pharmacol. 2012; 34(3):1004-13.

Ge S, Wang X, Xie J, Yi X, Liu F. Deep sequencing analysis of microRNA expression in porcine serum-induced hepatic fibrosis rats. Ann Hepatol. 2014; 13(4):439-49.

Hall C, Ehrlich L, Meng F, Invernizzi P, Bernuzzi F, Lairmore TC, et al. Inhibition of microRNA-24 increases liver fibrosis by enhanced menin expression in Mdr2-/- mice. J Surg Res. 2017; 217:160-169.

Hayes CN, Chayama K. MicroRNAs as Biomarkers for Liver Disease and Hepatocellular Carcinoma. Int J Mol Sci. 2016; 17(3):280.

He Y, Huang C, Sun X, Long XR, Lv XW, Li J. MicroRNA146a modulates TGF-betal-induced hepatic stellate cell proliferation by targeting SMAD4. Cell Signal. 2012; 24(10):1923-30.

Huang CF, Sun CC, Zhao F, Zhang YD, Li DJ. miR-33a levels in hepatic and serum after chronic HBV-induced fibrosis. J Gastroenterol. 2015; 50(4):480-90.

Jung YK, Yim HJ. Reversal of liver cirrhosis: current evidence and expectations. Korean J Intern Med. 2017; 32:213-228.

Kerr TA, Korenblat KM, Davidson NO. MicroRNAs and liver disease. Transl Res. 2011; 157(4):241-52.

Kitano M, Bloomston PM. Hepatic Stellate Cells and microRNAs in Pathogenesis of Liver Fibrosis. J Clin Med. 2016; 5(3).pii:E38. 
Krauskopf J, de Kok TM, Schomaker SJ, Gosink M, Burt DA, Chandler P, et al. Serum microRNA signatures as "liquid biopsies" for interrogating hepatotoxic mechanisms and liver pathogenesis in human. PLoS One. 2017; 12(5):e0177928.

Lakner AM, Steuerwald NM, Walling TL, Ghosh S, Li T, McKillop IH, et al. Inhibitory effects of microRNA 19b in hepatic stellate cell-mediated fibrogenesis. Hepatology. 2012; 56(1):300-10.

Leti F, Malenica I, Doshi M, Courtright A, Van Keuren-Jensen K, Legendre $\mathrm{C}$, et al. High-throughput sequencing reveals altered expression of hepatic microRNAs in nonalcoholic fatty liver disease-related fibrosis. Transl Res. 2015; 166(3):304-14.

Li M, He Y, Zhou Z, Ramirez T, Gao Y, Gao Y, et al. MicroRNA-223 ameliorates alcoholic liver injury by inhibiting the IL-6p47phox-oxidative stress pathway in neutrophils. Gut. 2017; 66(4):705715 .

Nakamura M, Kanda T, Jiang X, Haga Y, Takahashi K, Wu S, et al. Serum microRNA-122 and Wisteria floribunda agglutinin-positive Mac-2 binding protein are useful tools for liquid biopsy of the patients with hepatitis B virus and advanced liver fibrosis. PLoS One. 2017; 12(5):e0177302.

Ogawa T, Enomoto M, Fujii H, Sekiya Y, Yoshizato K, Ikeda K, et al. MicroRNA-221/222 upregulation indicates the activation of stellate cells and the progression of liver fibrosis. Gut 2012; 61(11):1600-9.

Okada H, Honda M, Campbell JS, Takegoshi K, Sakai Y, Yamashita T, et al. Inhibition of microRNA-214 ameliorates hepatic fibrosis and tumor incidence in platelet-derived growth factor $\mathrm{C}$ transgenic mice. Cancer Sci. 2015; 106(9):1143-52.

Roderburg C, Urban GW, Bettermann K, Vucur M, Zimmermann $\mathrm{H}$, Schmidt $\mathrm{S}$, et al. Micro-RNA profiling reveals a role for miR-29 in human and murine liver fibrosis. Hepatology. 2011; 53(1):209-18.

Roy S, Benz F, Vargas Cardenas D, Vucur M, Gautheron J, Schneider A, et al. miR-30c and miR-193 are a part of the TGF- $\beta$-dependent regulatory network controlling extracellular matrix genes in liver fibrosis. J Dig Dis. 2015; 16(9):513-24.

Schueller F, Roy S, Loosen SH, Alder J, Koppe C, Schneider AT, et al. miR-223 represents a biomarker in acute and chronic liver injury. Clin
Sci (Lond). 2017; 131(15):1971-87.

Shigehara K, Yokomuro S, Ishibashi O, Mizuguchi Y, Arima Y, Kawahigashi Y, et al. Real-time PCR-based analysis of the human bile microRNAome identifies miR-9 as a potential diagnostic biomarker for biliary tract cancer. PLoS One. 2011; 6(8):e23584.

Sun J, Zhang H, Li L, Yu L, Fu L. MicroRNA-9 limits hepatic fibrosis by suppressing the activation and proliferation of hepatic stellate cells by directly targeting MRP1/ABCC1. Oncol Rep. 2017; 37(3):16981706.

Trebicka J, Anadol E, Elfimova N, Strack I, Roggendorf M, Viazov S, et al. Hepatic and serum levels of miR-122 after chronic HCVinduced fibrosis. J Hepatol. 2013; 58(2):234-9.

Tsuchida T, Friedman SL. Mechanisms of hepatic stellate cell activation. Nat Rev Gastroenterol Hepatol. 2017; 14(7):397-411.

Udompap P, Kim D, Kim WR. Current and Future Burden of Chronic Nonmalignant Liver Disease. Clin Gastroenterol Hepatol. 2015; 13(12):2031-41.

Weber JA, Baxter DH, Zhang S, Huang DY, Huang KH, Lee MJ, et al. The microRNA spectrum in 12 body fluids. Clin Chem. 2010;5 6(11):1733-41.

Xu F, Liu C, Zhou D, Zhang L. TGF-B/SMAD Pathway and Its Regulation in Hepatic Fibrosis. J Histochem Cytochem. 2016; 64(3):15767

Yang X, Dan X, Men R, Ma L, Wen M, Peng Y, et al. MiR$142-3 p$ blocks TGF- $\beta$-induced activation of hepatic stellate cells through targeting TGFßRI. Life Sci. 2017; 187:22-30.

Zhai X, Cheng F, Ji L, Zhu X, Cao Q, Zhang Y, et al. Leptin reduces microRNA-122 level in hepatic stellate cells in vitro and in vivo. Mol Immunol. 2017; 92:68-75.

How to cite this article:

Ezhilarasan D. Role of MicroRNAs in Hepatic Fibrosis Progression. J App Pharm Sci, 2018; 8(05): 174-178. 